BULLETIN Bulletin hispanique

HISPANIQUE Université Michel de Montaigne Bordeaux

117-2 | 2015

Métamorphose(s) : représentations et réécritures

\title{
Métamorphoses et fragilité de l'homme et de ses sens dans les autos sacramentales caldéroniens
}

Yves Germain

\section{OpenEdition}

\section{Journals}

Édition électronique

URL : http://journals.openedition.org/bulletinhispanique/4004

DOI : 10.4000/bulletinhispanique.4004

ISSN : 1775-3821

Éditeur

Presses universitaires de Bordeaux

Édition imprimée

Date de publication : 15 décembre 2015

Pagination : 507-516

ISBN : 979-10-300-0041-2

ISSN : 0007-4640

Référence électronique

Yves Germain, « Métamorphoses et fragilité de l'homme et de ses sens dans les autos sacramentales caldéroniens », Bulletin hispanique [En ligne], 117-2 | 2015, mis en ligne le 15 décembre 2018, consulté le 01 mai 2019. URL : http://journals.openedition.org/bulletinhispanique/4004 ; DOI : 10.4000/ bulletinhispanique.4004 


\title{
Métamorphoses et fragilité de l'homme et de ses sens dans les autos sacramentales caldéroniens
}

\author{
Yves Germain \\ Université de Paris-Sorbonne
}

L'article envisage les limites et les modalités d'une représentation des métamorphoses du corps humain dans les " autos " caldéroniens. Une telle représentation, contraire à la finalité du genre, la célébration de l'Eucharistie et donc du corps du Christ sauveur, apparaît comme une tentation récurrente de la dramaturgie, qui se manifeste notamment dans les allégories des sens, et pourrait trouver une justification dans la réflexion augustinienne sur les métamorphoses.

Mots-clés : Calderón, Auto sacramental, métamorphoses, sens.

El artículo considera los límites y las modalidades de una representación de las metamorfosis del cuerpo humano en los autos calderonianos. Esta representación, contraria a la finalidad del género, la celebración de la Eucaristía y así del cuerpo de Cristo, surge varias veces como una tentación de la dramaturgia, como en las alegorías de los sentidos, y podría hallar alguna justificación en la reflexión agustiniana sobre las metamorfosis.

Palabras-claves : Calderón, Auto sacramental, metamorfosis, sentidos.

This paper envisages the limits and modes in representing the human body's metamorphoses in Calderón's «autos». Such representation, contrary to the purpose of the genre -i.e. the celebration of the Eucharist and of Christ's body- nevertheless appears as a recurring temptation for the dramatist, in particular through the allegory of the senses, and could find some justification in the Augustinian thought about metamorphoses.

Keywords : Calderón, Auto sacramental, metamorphosis, senses. 
C I la FAscination de la métamorphose, le goût pour les figures protéiformes $\checkmark$ du change, tiennent une place primordiale au sein du spectacle baroque, comme l'avait si bien montré Jean Rousset, l'auto sacramental tel que Calderón le consacre, constitue-t-il un mode d'expression propice à leur étude ? Sans doute célèbre-t-il, avant tout, la plus étrange des métamorphoses, cette transsubstantiation qui, pour demeurer inéluctablement opaque aux hérétiques, ne doit pas moins paraître au public comme une évidence du dogme, un mystère sacré qu'on ne s'aventurera pas à faire entrer dans le champ d'étude des métamorphoses ; ce mystère central du merveilleux que l'auto véhicule ne saurait toutefois être éludé pour son impact sur le spectacle, par la contrainte idéologique qu'il impose aux formes du surnaturel, comme on va le voir. Pour le reste, le genre de l'auto offre une étonnante combinaison de fixité et de souplesse. La fixité, dans le message comme dans la forme, tient en un millier de vers que se partagent des figures surtout allégoriques, aux rôles prédéfinis pour les besoins de l'édification, suivant une trame tendue vers la célébration de l'Eucharistie selon une règle confinant presque à une liturgie. Le spectacle de la métamorphose pourrait-il trouver sa place sur ce théâtre-là ? L'autre caractéristique du genre, l'étonnante souplesse que Calderón lui confère en lui incorporant de multiples apports de la comedia et du théâtre de cour, selon un jeu constant de variations, pourrait bien le faire penser. Le spectacle du merveilleux tend à s'y déployer de plus en plus, sous l'influence des modes du palais, pour affecter les mutations de l'espace scénique, dans la richesse des décors mobiles, voire pour frapper les esprits par des effets spectaculaires de la puissance des forces du Bien.

Mais si le merveilleux semble adaptable aux possibilités d'une scène qui s'offre la liberté de ses audaces techniques, en va-t-il de même pour les métamorphoses du corps que pour les transformations de l'espace?

Le corps dans sa fondamentale fragilité, sa faiblesse exposée aux tentations du démon, est une thématique récurrente des autos caldéroniens, que ce théâtre allégorique décline à travers le motif de la défaillance presque inéluctable des sens. Le groupe animé et plaisant des cinq sens n'a cessé de renforcer sa place sur la scène des autos, pour devenir dans ceux de la maturité une composante presque obligée de l'auto ou de la loa qui l'introduit. Le jeu de l'homme et de ses sens s'offre comme un des ingrédients les plus adaptés à la mise en œuvre du spectacle, susceptible souvent d'agrémenter l'exposé doctrinal par les intermèdes plaisants auxquels il se prête : dans cette optique, on pourrait le croire propice aux manifestations de la métamorphose. L'analyse des passages mettant en scène le corps et ses sens fait apparaître une configuration plus complexe et, semble-t-il, résolument contrainte. On examinera d'abord le motif presque obsessionnel de la stupeur qui suspend l'usage des sens, figurant une première transformation qui semble figer l'homme en statue. Cette figure du discours ne devient qu'exceptionnellement manifestation scénique, avec la métamorphose de l'homme en statue comme œuvre du démon, comme on le verra dans El diablo mudo. On examinera ensuite comment le groupe allégorique des cinq sens se prête de façon plus spectaculaire, à l'occasion, à un jeu de 
métamorphoses animales, dans deux autos mettant en scène les enchantements d'une magicienne : Aurora Egido l'avait étudié, dans un essai désormais classique, dans Los encantos de la culpa ${ }^{1}$; en en lisant l'écho plus tardif dans $E l$ jardin de Falerina, on mesure que cette métamorphose des sens, toujours objet réjouissant du discours, peut aussi être un ingrédient, assez restreint néanmoins, du spectacle. On pourra donc, dans un troisième temps, s'interroger sur ces résistances à figurer la métamorphose des corps, dans un genre pourtant si ouvert au merveilleux. La contrainte appliquée aux manifestations pourtant si tentantes de la métamorphose, pleines de séduction pour le public, n’a rien de fortuit. La célébration du corps du Christ à travers l'Eucharistie imposait une limite radicale à la représentation des métamorphoses corporelles, et la fixité idéologique prenait le pas sur la souplesse théâtrale du genre.

Avant de nous interroger, pour cet ouvrage sur les métamorphoses du corps, un précédent colloque sur le silence, à Paris-Sorbonne ${ }^{2}$, avait attiré notre attention vers une forme de bouleversement qui saisit assez fréquemment la figure de l'homme dans les autos caldéroniens : un trouble soudain, affectant l'ensemble de ses sens en plus de sa voix, un saisissement, une stupeur qui suspend leur usage. Ce trouble reflète tout d'abord un lieu commun du discours caldéronien, qui ne se limite ni aux autos ni à la figure humaine; dans les autos, cette diction d'un mutisme imposé par la stupeur affecte aussi les puissances du mal, le démon ou la faute, quand leur action est battue en brèche. Mais la place cruciale accordée aux sens, et à la fragilité à laquelle ils exposent l'homme en proie aux tentations du péché, fait que cette stupeur s'impose, notamment dans les autos de la maturité, comme un phénomène marquant affectant la figure humaine, une transformation de son être au monde. La répétition de la chute peut ainsi entraîner un évanouissement, comme dans La nave del Mercader (1674), après que l'homme a bu la coupe que lui tendaient le Démon et la Luxure (Lascivia) : tandis qu'un «terremoto » se fait entendre, les figures présentes commentent en chantant "que hombre en pecado, no sólo bruto es, que no discurre / pero idolo inmóvil, que ni hable, ni escuche, / ni vea, ni toque, ni huela, ni guste " ${ }^{3}$. L'idole immobile ne l'est cette fois que dans le discours, et l'homme doit alors bientôt se réveiller pour rendre des comptes aux créanciers que sont devenus ses sens et les puissances de l'âme, qui le conduiront à un cachot d'où sa plainte pourra s'élever vers le Ciel. Le mutisme est alors bref et de pure forme, tandis que ce motif de la transformation en idole, la

1. Aurora Egido, La fábrica de un auto sacramental : "Los encantos de la culpa ", Salamanca, Universidad de Salamanca, 1982 ; repris dans A. Egido, El gran teatro de Calderón. Personajes, temas, escenografía, Kassel, Reichenberger, 1995, et dans Javier Aparicio Maydeu, Estudios sobre Calderón, t. 2, Madrid, Istmo, 2000.

2. Il s'agit du colloque Les voies du silence dans l'Espagne des Habsbourg, en 2008, dont les actes, édités par les organisatrices Araceli Guillaume et Alexandra Merle, ont parus aux PUPS en 2013 ; voir notre article " La question du silence dans les autos caldéroniens, ou l'impossible mutisme ", p. 243-255.

3. Toutes nos citations renvoient à l'édition des autos en un seul volume, dans le t. 3 des Obras completas de Calderón, Madrid, Aguilar, 2e éd., 1991, p. 1462. 
pétrification implicite, laisse voir une source des Psaumes, parfois mentionnée, de cette stupeur muette qui suspend les sens. Il s'agit du Psaume 134 (15-18), qui ne décrit pas l'homme en état de péché mais les idoles des païens :

Elles ont bien une bouche, mais elles ne soufflent mot, Elles ont bien des yeux mais elles n'y voient rien; des oreilles, mais elles n'entendent rien. Et pas le moindre souffle dans leur bouche! Que ceux qui les ont fabriquées deviennent comme elles, et quiconque aussi met sa confiance en elles !

Cette source est bien évidemment susceptible de mener à une métamorphose de l'homme en statue, qui explore l'effet scénique du discours. L'adaptation en auto d'une zarzuela en deux actes consacrée aux enchantements de la magicienne Falerina, inspirée de l'Arioste, El jardín de Falerina (1675), aurait pu le permettre. La transformation en statue de Roger / Rugero, puis de toute la troupe de Charlemagne qui tentait de le délivrer (à l'exception de Roland, qui fait cesser l'enchantement) était l'ingrédient spectaculaire de cette zarzuela plus ancienne (1640 ?). Or dans l'auto, la Faute, avatar de Falerina et " falaz sirena ", ne parvient à produire qu'une pétrification très partielle de l'homme, lorsqu'il boit la coupe qu'elle lui tend. Hombre a beau dire que ce "letargo mortal " le prive de sa parole et de ses sens, il continue de s'exprimer et de commenter les effets du mal :

\author{
Estatua viva soy \\ Pues tengo (;ay infeliz!) \\ Ojos para no ver, \\ Oídos para no oír, \\ Labios para no hablar \\ Plantas para no huir, \\ Para no tocar manos $[\ldots]^{4}$
}

suscitant chez la Faute le rappel de la source «Así, en ídolos de bronce / te definió David ». L'adaptation se heurte donc à une difficulté, celle de concrétiser par un jeu scénique prolongé la suspension de l'humanité vivante et sa pétrification. Un seul auto apparemment y était parvenu, El diablo mudo, de 1660. L'oxymore du titre - alors que le diable est surtout coutumier d'une parole facile - ne renvoie qu'au dénouement, quand le Salut chrétien, opéré par Peregrino, vient abolir l'insidieuse mise en scène orchestrée par le Démon. C'est ce dernier en effet qui prive l'homme de sa parole et de ses sens, une transformation effective que l'homme lui-même n'est plus en mesure de gloser :

\author{
esto es el Hombre en pecado \\ siendo el idolo que cuenta \\ allá el salmista; pues tiene \\ ojos y no mira; lengua
}

4. Éd. cit., p. 1517. 


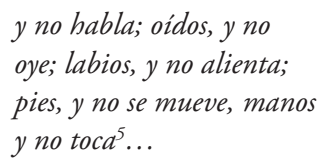

Le "vivo cadáver", s'il a perdu la parole, peut encore parfois se mouvoir, voire s'agiter comme un possédé qui suscite des commentaires parfois amusés (" habla más por señas / que un garito de barberos, I un soportal de ropero / y una antesala de dueñas."). Cette fausse statue qui ne commande rien n'est plus vraiment humaine, à la satisfaction du Démon qui voit dans son œuvre " un hombre en forma de fiera / o una fiera, que es más cierto / en forma de hombre del monte ", un sauvage "haciendo extremos". Le dénouement, avec une transposition de la Passion qui charge particulièrement la figure du Judaïsme, permettra que l'Homme retrouve son être et sa santé.

Cet auto n'eut pas de succès : un échec peut-être imputable à un changement de compagnie lors de sa représentation ${ }^{6}$, mais auquel on peut supposer des motifs plus conséquents, qui tenaient à la représentation par trop déformée de l'homme et de son corps, éventuellement mise en évidence par un jeu d'acteur maladroit. Le mutisme réel heurte sans doute la logique d'un genre allégorique où toute figure doit pouvoir s'expliquer; mais plus encore cette fausse statue, qui ne s'anime que pour des pitreries dignes d'un possédé, renvoyait à coup sûr une image trop dégradée de l'homme. Ce piètre avatar de la figure humaine, qui ne pouvait être que l'œuvre d'un Démon metteur en scène d'un théâtre dans le théâtre, était sans doute parvenu à déranger le public. Calderón ne renouvellera pas dans ses autos cet unique essai un peu prolongé de métamorphose de l'homme en statue muette.

Le jeu des cinq sens lui fournissait, il est vrai, de multiples possibilités scéniques que leur nature d'allégories distinctes de celle de la nature humaine rendait plus libres, autorisant à l'occasion des métamorphoses animales forcément divertissantes. Les cinq sens assurent en effet le passage de l'allégorie morale à la scène de comédie, sans jamais cesser de s'intégrer au propos didactique. Leurs querelles de préséance tournent à la dispute de courtisans vexés, dans El nuevo Palacio del Retiro, où l'Homme qui est aussi le valido, doit intervenir quand ils en viennent à tirer l'épée ; leur procès contrastif, quant à leur perception de l'Eucharistie, est souvent instruit par la Foi, toujours au bénéfice de l'Ouïe, le plus spirituel des cinq sens, qui seul saisit la valeur de l'hostie là où les quatre autres ne voient, sentent, touchent ou goûtent que du pain. La confrontation de ce groupe animé aux maléfices d'une magicienne qui mettra en évidence la fragilité qu'ils confèrent à l'Homme, est dans ces conditions susceptible d'inspirer des métamorphoses plaisantes de ces cinq figures.

Le mythe d'Ulysse et de Circé s'y prêtait tout particulièrement, en offrant

5. Ibid., p. 946.

6. Voir l'introduction de Celsa Carmen García Valdés à son édition de l'auto pour la collection des Autos sacramentales completos ( $\mathrm{n}^{\circ}$ 26), Kassel, Reichenberger, Pamplona, Universidad de Navarra, 1999, p. 9-11. 
la possibilité de faire de ces sens les compagnons de l'Homme-Ulysse, que l'enchanteresse, devenue la Faute, métamorphose en bêtes après que les Vices, ses dames de compagnie, les ont séduits chacun par leur point faible. Saint Augustin, dans La Cité de Dieu (XVIII, 17) avait brièvement évoqué " maga illa famosissima Circe quae socios quoque Ulixis mutavit in bestias ", comme exemple fameux des métamorphoses dans ce merveilleux païen sur lequel il s'interroge (sans légitimer pour autant l'usage allégorique de l'épisode, comme on le lit parfois). Calderón s'en souvient dans Los encantos de la culpa, en 1645, adaptant ainsi comme auto sa première pièce mythologique jouée au Buen Retiro en 1635, El mayor encanto, Amor : on notera cependant que la métamorphose animale y reste un effet de discours, raconté par l'Entendement, alors que la comedia rendait les figures animales présentes sur scène. Son récit des transformations établit un système analogique, montrant l'action d'un vice sur un sens donné, et la métamorphose de ce dernier en vertu d'une explication tenant de l'énigme ou du mot d'esprit. La vue est ainsi transformée par l'envie en " tigre " - plutôt une panthère - (" que este animal todo es ojos ") ; l'ouïe l'est en caméléon sous l'influence de la flatterie ; l'odorat relié à la médisance devient de façon plus obscure un lion. Le toucher séduit par la luxure est alors un ours, tandis que le goût mené par la gourmandise ne peut être qu'un porc, "bruto inmundo" qu'Entendement répugne même à nommer ${ }^{7}$. Ce bestiaire ne s'anime ensuite, et très partiellement, que dans le souvenir des sens réveillés par l'intervention de l'Homme, qui remporte sur la Faute une première victoire avant de lui céder ensuite : tous répugnent à ce réveil, comme à reprendre la mer, et le goût, gracioso de la compagnie, se complaît au souvenir du porc qu'il était dans une sorte de rêve, insistant sur cette existence paisible et repue : "No sabes cuán bueno es / estar comiendo y gruñendo? ". Le motif même de l'animalité s'efface ensuite, alors que les sens ne font qu'accompagner l'Homme séduit par l'espace enchanteur du jardin-palais.

Aussi la reprise de la métamorphose animale des sens dans Eljardin de Falerina (1675) s'avère-t-elle plus riche, dans la mesure où elle apparaît effectivement sur scène. Cette fois, la mythologie moderne dérivée de l'Arioste remplace le mythe de Circé, mais concernant les sens, Calderón se livre à un réemploi qui ne le gênait nullement, trente ans après Los encantos de la culpa. La tirade des métamorphoses est cette fois prononcée par la Faute elle-même, qui rend compte de la transformation opérée sur les sens par les vices, ses cinq formes, dans son jardin enchanté. Les trois animaux repris sont évoqués en premier : le tigre pour la vue, le caméléon pour l'ouïe et le lion pour l'odorat, en emblèmes respectifs de l'envie, de la flatterie (car le caméléon est censé ne se nourrir que d'air), de la médisance (le souffle du lion "daña cuanto encuentra ") : la réécriture, plus concentrée, est aussi plus claire. On ne la glosera pas ici, puisque Aurora Egido avait étudié ces premières associations à propos de Los encantos de

\footnotetext{
7. Éd. cit., p. 411.

8. Ibid., p. 414.

9. Ibid., p. 1520.
} 
la culpa avec le soin savant qu'on lui connaît. Les deux sens restants changent de figure animale, alors que l'ours et le porc, si chers à Michel Pastoureau, semblaient s'associer aisément au toucher et au goût ${ }^{10}$ : mais il s'agit aussi bien de surprendre que de trouver une nouvelle façon de faire sourire.

Le toucher est ainsi transformé par la luxure " en un torpe erizo", animal que nul ne peut toucher, métaphore ainsi de sa propre paralysie. Le goût, comme dans la tirade antérieure de l'Entendement, n'est pas identifié sur-le-champ et se donne donc en devinette : "la voraz bestia, que ásperamente cerdosa / no levanta de la Tierra / los ojos al Cielo, y sólo / de lo inmundo se alimenta ». La malheureuse bête ainsi érigée en icône du matérialisme et d'une gloutonnerie qui se complaît dans l'immonde est plus loin identifiée par une didascalie comme un "espin ", un porc-épic! L'ultime avatar des pourceaux de Circé dans L'Odyssée (seul animal alors de la métamorphose des compagnons d'Ulysse) emprunte au hérisson ses piquants peu engageants; tous deux pourraient procéder du bestiaire des livres d'emblèmes, qui en font un certain usage et tendent parfois à les confondre, comme le fait Saavedra Fajardo ou plutôt son illustrateur, dans les emblèmes 59 et 82 des Empresas políticas (dans l'édition milanaise de 1642, éditée par Sagrario López ${ }^{11}$ ), lorsqu'il utilise comme symbole des armes dressées (« sus púas, con las cuales parecen cerrado escuadrón de picas") "un espin " ressemblant fort à un hérisson. Le sérieux des armes a toutefois entièrement disparu ici, où le porc chargé de piquants est foncièrement burlesque et doit trouver une fonction scénique ; et de fait, c'est dans le théâtre burlesque que nous avons trouvé une autre mention de l'"espin ", bien loin de Saavedra Fajardo : dans l'entremés de Los putos, de Jerónimo de Cáncer, le gracioso Toribio s’identifierait volontiers à cet animal repoussant, lorsqu'une lettre enchantée d'abord conçue pour lui attribuer les grâces de Menga, suscite à son égard l'enthousiasme amoureux d'un sacristain, d'un greffier et d'un alguazil :

$$
\begin{aligned}
& \text { — ¡Ay qué hermosos pies y piernas! } \\
& \text { —iqué barbas! } \\
& \text { TORIBIO.— ¿de puerco espin! }{ }^{12}
\end{aligned}
$$

Le goût mué en porc-épic, dans l'auto, ne sort donc pas du registre du gracioso, et le discours de la Faute prépare l'apparition sur scène de costumes divertissants. Et, de fait, si dans un premier temps, on voit apparaître, dans une didascalie, autour de l'homme " a manera de estatua", "un león, un tigre, un espin, un erizo y un camaleón ", ce bestiaire s'anime juste avant le dénouement, lorsque les sens, délivrés de leur torpeur, surgissent sur scène " en sus trajes, luchando con los cinco vicios $»^{13}$.

10. Michel Pastoureau, Lours, histoire d’un roi déchu, Paris, Seuil, 2007 et Le cochon, histoire dun cousin mal aimé, Paris, Gallimard, coll. « Découvertes », 2009.

11. Madrid, Cátedra, coll. "Letras hispánicas " (n 455), 1999, p. 685 (emblema 59) et p. 983 (emblema 82).

12. Antología del teatro breve español del siglo XVII, éd. Javier Huerta Calvo, Madrid, Biblioteca nueva, 1999, p. 271-272.

13. Obras completas, t. 3, éd. cit., p. 1524. 
L'animation zoomorphe se renforce ici par rapport à la zarzuela-source, où l'enchanteresse Falerina, lorsqu'elle transforme Rugero en statue, ordonne aux deux graciosos couards du premier acte, le Français Jaques et le maure Zulemilla, qui reparaissent vêtus en lions, de veiller sur lui. Jaques, pour se dérober, fait à nouveau entendre sa nature burlesque, en démentant son apparence de lion " aunque león parezca, soy puerco, y aun espin, ¿cómo he de defenderle? " ${ }^{14}$. Cet espin-là, bien loin de la littérature emblématique, est plus susceptible de se mettre en boule que de figurer une force armée ; Jaques, lion bien dérisoire, continue de n'écouter que sa lâcheté, mais sa réplique, par la mention isolée et très inattendue de la bête à piquants, laisse deviner une raison probable de l'insolite passage du lion au porc-épic : il n'y avait pas loin, peut-on penser, de la crinière léonine aux piquants dans le costume de scène de ce Jaques, et l'on est dès lors moins surpris de voir côte à côte dans l'auto, la curieuse série lion/porc-épic/hérisson, unie par des costumes offrant un probable dégradé de fourrure et de piquants. Ce bestiaire hérissé reparaît donc dans la scène finale de l'auto, pour une scène d'action brève mais certainement spectaculaire : alors qu'un tremblement de terre résonne, bousculant le palais de la Faute, les cinq vices se lamentent et les cinq sens crient leur joie, et se libèrent de leur emprise.

\begin{tabular}{|c|c|}
\hline Les vices: & ¿Qué ansia, qué dolor, qué pena! \\
\hline Les sens : & iqué dicha y qué gozo! \\
\hline Les vices: & - oye - aguarda - jescucha! - jespera! \\
\hline La vue: & - Envidia, ;quita! \\
\hline L'ouïe : & - Lisonja, japart \\
\hline Le toucher : & - Lascivia, jsuelta! \\
\hline e goût : & - iNo, Gula, a mí toques! $!^{15}$ \\
\hline
\end{tabular}

On suppose ces deux derniers bien convaincants avec leurs piquants et l'on retrouve la syntaxe incorrecte du goût, copiée sur celle des deux graciosos étrangers de la zarzuela. Sans doute, l'intermède burlesque est-il bref, mais il ajoute une note supplémentaire à la défaite de la Faute, là où Los encantos de la culpa éludait cette possibilité plaisante pour insister davantage sur la métamorphose de l'espace, avec la disparition du palais de Circé. Cette fois, les sens réveillés de l'enchantement, peuvent user de leurs déguisements pour faire rire de la métamorphose passée en la rejouant sur scène.

Bien petite moisson, dira-t-on, que ces quelques métamorphoses animales et leur réalisation scénique encore plus restreinte, en regard du corpus si vaste des autos... Elle témoigne au moins, à trente ans d'écart entre Los encantos et $E l$ jardín de Falerina, de la reprise d'une idée suggestive, extension spirituelle de la pensée analogique des autos, et, dans la curieuse apparition du porc-épic, d'un détail révélateur de l'attention de Calderón à la matérialité du spectacle, dans

14. Calderón, Obras completas, éd. Ángel Valbuena Briones, Madrid, Aguilar, 1956, t. 2, p. 1908.

15. Obras completas, t. 3, éd. cit., p. 1525. 
un souci de réemploi dans l'auto des ingrédients de tout le théâtre du temps. Une piètre crinière de faux lion reparaît comme dos hérissé d'un porc-épic, et l'un comme l'autre se mêlent en un bestiaire improbable qu'on rêve de remettre en scène.

La possibilité restreinte des métamorphoses de l'homme et de ses sens, pourtant figures centrales d'un genre où la mise en scène multiplie les changements et métamorphoses de l'espace, et ne dédaigne pas de recourir aux effets spectaculaires du merveilleux, pourrait a priori surprendre. Elle est pourtant l'effet logique d'un cadre de pensée augustinienne de la métamorphose, parfaitement illustré par ce qu'on vient de voir, et, plus encore, dans un genre voué à la célébration du Corpus et de l'Eucharistie, la conséquence inévitable d'une vision du corps humain, dont le Christ est comme le garant, par le sacrifice de son propre corps pour le Salut des hommes.

Le cadre largement augustinien de la pensée caldéronienne n'interdit certes pas l'intérêt pour les métamorphoses qui alimentaient les fables des Anciens et de leurs poètes : Augustin leur consacre une partie du livre XVIII de sa Cité de Dieu, mentionnant l'attrait suscité par l'histoire de Circé, les récits de lycanthropie, la métamorphose en oiseaux des compagnons de Diomède, chantée par Ovide (Métamorphoses, XIV), ou bien encore l'histoire de L'Âne dor, d'Apulée (Civ. Dei, XVIII, 16-18). Il n'est pas moins intrigué par ces histoires de femmes aubergistes qui servent aux voyageurs, dans leur fromage, de quoi les transformer en bêtes de somme en fonction de leurs besoins du moment. Sa réponse face à de tels mystères tient à l'œuvre des démons qui, transformant seulement l'apparence des créatures créées par Dieu, et non leur être, suscitent des illusions « de sorte qu'elles paraissent être ce qu'elles ne sont pas ${ }^{16}$.

Illusions qui sont l'œuvre des démons, voilà bien ce que sont les métamorphoses représentées dans les autos étudiés plus haut : on se souvient du Démon metteur en scène de l'homme privé de parole et pétrifié dans El diablo mudo, où la transformation, imparfaite, est bien jeu d'apparences, illusion qui suscite l'étonnement de l'assistance mais ne parvient pas à changer l'être de l'homme, qui ne prend l'apparence d'une statue encore assez mobile que le temps de cette mise en scène. Quant à la possibilité de la métamorphose du corps de l'homme en figure animale, si Augustin la rejette, il conçoit que cette illusion, semblable à celles que produit le sommeil, soit susceptible d'opérer " une fois les sens corporels assoupis ou inhibés ${ }^{17}$ : c'est précisément là le biais que les enchanteresses caldéroniennes, Circé ou Falerina, figures du démon, mettent à profit dans leurs jardins magiques. Assoupir les sens (comme Circé qui les plonge dans un "sueño ${ }^{18}$ ) ou les inhiber par l'entremise des vices,

16. La Cité de Dieu, XVIII, 18, dans Euvres, éd. Lucien Jerphagnon, Paris, Gallimard, coll. "La Pléiade", t. 2, p. 784.

17. Ibid., p. 784.

18. Sur cette notion, voir l'article de Françoise Gilbert, « Sobre sueños y visiones en el auto de Calderón Los encantos de la culpa", dans Ignacio Arellano et Dominique Reyre (sous la dir. de) El mundo maravilloso de los autos de Calderón, Pamplona-Kassel, Universidad de Navarra, 
pour produire l'illusion des métamorphoses animales : illusion que rapporte l'Entendement dans Los encantos de la culpa, et qu'Ulysse dissipe en réveillant les sens contre leur gré ; effet dont se vante la Faute dans Falerina, mais qui restera une apparence, effet de discours d'abord, puis simple costume de scène quand à la fin les sens se libèrent de l'emprise des vices.

Encore la métamorphose animale des sens, dans ces deux autos, est-elle facilitée par le fait que leurs allégories, au sens strict, les font attributs de l'homme mais ne sauraient les associer à la figure humaine conçue comme unité. Or, dans un genre voué par son contexte - la fête du Corpus - et sa finalité - la célébration de l'Eucharistie - à célébrer le corps du Christ, il doit être vain d'espérer une représentation d'une métamorphose du corps humain. Même accablé par la faiblesse de ses sens et voué à la répétition du péché, le corps humain reste à l'image de son sauveur qui s'est incarné pour assurer son salut. Du moment où l'auto doit célébrer la communion dans le corps du Christ, l'intégrité du corps de l'homme ne saurait être altérée. L'altération de ses sens ou le mutisme peuvent bien survenir comme effets de la stupeur, pour un temps; ils ne sauraient mener à la transformation de l'homme en statue, en cette idole évoquée par la référence au Psaume 134. L'idole à laquelle est vouée l'idolâtre, ou la statue de sel que devient la femme de Loth, restent des images de l'Ancien Testament et le destin de pierre de ceux qui ont renoncé au salut. L'humanité chrétienne des autos résiste à toute métamorphose, au nom de la finalité du genre.

On aurait pu, à coup sûr, inverser la démarche de ce travail ; constatant une impossibilité, il eût peut-être été préférable de le faire jusqu'à en esquiver la matière même : un tel sujet n'aurait tout simplement pas lieu d'être. La réalité de la pratique théâtrale de Calderón dément néanmoins cette rigueur : le souci $\mathrm{du}$ dramaturge est d'apporter au genre cette indispensable variation qui lui permet de ne pas le figer, d'y incorporer autant que possible les ingrédients du spectacle théâtral extérieur à l'auto pour retenir l'intérêt du public. Or la métamorphose des figures de la scène opère dans l'imaginaire du temps une profonde séduction, et la tentation de l'introduire est grande. Le discours la suggère à l'envi dans les propos de l'homme défait par le péché, atteint par la défaillance de ses sens, si la dramaturgie à visée eucharistique l'interdit. Et le réemploi des métamorphoses animales dont le théâtre de cour a fait son miel avec les histoires de Circé et de Falerina, grâce à la diversification allégorique des sens, déploie dans le discours son onirisme païen, joue sur le trouble des rêves et des illusions que les magiciennes autorisent, et, à l'occasion, surgit comme un jeu de scène burlesque, où l'excès assumé du déguisement, réduit à sa matérialité scénique, permet l'audacieux désordre. 